\title{
Emotion-induced blindness reflects competition at early and late processing stages: An ERP study
}

\author{
Briana L. Kennedy • Jennifer Rawding • Steven B. Most • \\ James E. Hoffman
}

(C) Psychonomic Society, Inc. 2014

\begin{abstract}
Emotion-induced blindness (EIB) refers to impaired awareness of items appearing soon after an irrelevant, emotionally arousing stimulus. Superficially, EIB appears to be similar to the attentional blink (AB), a failure to report a target that closely follows another relevant target. Previous studies of $\mathrm{AB}$ using event-related potentials suggest that the $\mathrm{AB}$ results from interference with selection ( $\mathrm{N} 2$ component) and consolidation ( $\mathrm{P} 3 \mathrm{~b}$ component) of the second target into working memory. The present study applied a similar analysis to EIB and, similarly, found that an irrelevant emotional distractor suppressed the $\mathrm{N} 2$ and $\mathrm{P} 3 \mathrm{~b}$ components associated with the following target at short lags. Emotional distractors also elicited a positive deflection that appeared to be similar to the $\mathrm{P}_{\mathrm{D}}$ component, which has been associated with attempts to suppress salient, irrelevant distractors (Kiss, Grubert, Petersen, \& Eimer, 2012; Sawaki, Geng, \& Luck, 2012; Sawaki \& Luck, 2010). These results suggest that irrelevant emotional pictures gain access to working memory, even when observers are attempting to ignore them and, like the $\mathrm{AB}$, prevent access of a closely following target.
\end{abstract}

Keywords Emotion $\cdot$ Attention $\cdot$ ERP

\section{B. L. Kennedy $(\square) \cdot$ S. B. Most}

School of Psychology, The University of New South Wales, Sydney, NSW 2052, Australia

e-mail: b.kennedy@student.unsw.edu.au

S. B. Most

e-mail: s.most@unsw.edu.au

J. Rawding

Princeton University, Princeton, NJ 08544, USA

S. B. Most $\cdot$ J. E. Hoffman

Department of Psychology, University of Delaware, Newark, DE 19716-2577, USA

J. E. Hoffman

e-mail: hoffman@psych.udel.edu

\section{Introduction}

Emotionally arousing stimuli can impair visual awareness of items presented a short time later-an effect known as emotion-induced blindness (EIB; Most, Chun, Widders, \& Zald, 2005; Wang, Kennedy, \& Most, 2012). In a typical EIB task, participants search for a single target picture embedded in a series of rapidly presented pictures. When the target is preceded at short intervals by a task-irrelevant emotional distractor, accuracy in reporting the target is impaired. What mechanisms underlie this impairment? Are the targets suppressed early in perceptual processing? Or do they receive extensive perceptual and semantic processing, failing to reach awareness due to later bottlenecks such as consolidation into working memory (WM)?

At least superficially, EIB is reminiscent of the attentional blink (AB), a phenomenon characterized by impaired awareness of the second of two targets (T2) embedded in a rapid stream of stimuli when it appears shortly after the first target (T1). One influential theory of the $A B$ postulates that visual awareness in this context emerges via two stages of processing: an initial high capacity stage responsible for producing a perceptual and semantic representation of the object and a second, capacity-limited, serial stage in which this representation is consolidated into WM (Chun \& Potter, 1995). If the second target appears while the first target is still being consolidated, it must queue for stage 2 resources and remains vulnerable to decay or masking by subsequent items in the stream (Dell'Acqua, Jolicoeur, Luria, \& Pluchino, 2009; Dux, Asplund, \& Marois, 2009; but see also Olivers, van der Stigchel, \& Hulleman, 2007). Variants of this theory fall under the rubric of a central interference theory to underscore the claim that blinked items are fully processed through perceptual and semantic levels and are missed because of interference at the level of WM consolidation (Vogel, Luck, \& Shapiro, 1998). 
Neuroimaging studies have provided support for the central interference theory of the AB. In particular, methods with high temporal resolution, such as the event-related brain potential (ERP) and magnetoencephalography (MEG), are capable of providing insight into the rapid mental processes involved in responding to rapid serial visual presentations of objects in AB and EIB studies. Vogel et al. (1998) examined several ERP components elicited by the second target in an $\mathrm{AB}$ task and found that the $\mathrm{P} 3 \mathrm{~b}$, which they suggested reflects consolidation of information into WM (e.g., Vogel \& Luck, 2002), is suppressed during the blink. In contrast, several ERP components were unaffected by the blink, including the P1 and N1 components, reflecting early perceptual and attentional processes, as well as the N400, reflecting activation of semantic information (e.g., Vogel et al., 1998; see also Luck, Woodman, \& Vogel, 2000; Vogel \& Luck, 2002). These results are consistent with a central locus of interference, since they suggest that processes involved in identification and semantic activation - thought to be the province of stage 1-remain intact during the $\mathrm{AB}$, whereas stage 2 processes involving consolidation of information into WM, reflected by the $\mathrm{P} 3 \mathrm{~b}$ component, are suppressed.

The notion that the $\mathrm{AB}$ stems from competition for a limited-capacity system such as WM consolidation yields the prediction that there should be a trade-off in the amplitude of the P3bs associated with $\mathrm{T} 1$ and $\mathrm{T} 2$. In other words, a large P3b elicited by $\mathrm{T} 1$ (reflecting access to the limited-capacity system) should be accompanied by a small P3b to T2, reflecting a reduction in the degree to which $\mathrm{T} 2$ accesses this system. Furthermore, this trade-off should be mirrored by a similar pattern in the accuracy of reporting T1 and T2. Consistent with such predictions, this pattern of results emerged from an AB study that employed MEG (Shapiro, Schmitz, Martens, Hommel, \& Schnitzler, 2006). When the targets were separated by a large, 600-ms interval, participants were able to identify both targets, and both targets elicited a large M300 (the MEG equivalent of the P3b). However, when T2 appeared $200 \mathrm{~ms}$ after T1, detection of T2 was reduced, and failures to report T2 were accompanied by both an enhanced M300 for T1 and a reduced M300 for T2. However, when T2 was correctly reported, the M300 for T1 was small, and the M300 for T2 was enhanced. This evidence is consistent with the claim that the P3b/M300 reflects a limited capacity resource involved in the $\mathrm{AB}$ and underscores the potential role of $\mathrm{WM}$ consolidation processes in the $\mathrm{AB}$.

Additional evidence suggests that a component that may be related to selection of the second target is also suppressed in the blink. Sergent, Baillet, and Deheane (2005) reported that both the $\mathrm{P} 3 \mathrm{~b}$ and the earlier, posterior $\mathrm{N} 2$ components (latency of $270 \mathrm{~ms}$ ) associated with $\mathrm{T} 2$ were suppressed during the blink, a finding that has been replicated several times (Kranczioch, Debener, Maye, \& Engel, 2007; Reiss, Hoffman, Heyward, Doran, \& Most, 2008). The posterior
$\mathrm{N} 2$ observed in $\mathrm{AB}$ might be related to the N2pc component, which indexes selection of an object in the presence of distractors (Woodman, Arita, \& Luck, 2009). Sergent et al. noted that the N2-P3b sequence of components may reflect sequential stages in the operation of a single, limited-capacity system. This system might be responsible for selecting a relevant object from distractors and consolidating it into WM.

The phenomenological similarity between EIB and the $\mathrm{AB}$ raises the possibility that we might observe the same pattern of ERP components during EIB as occur during the $\mathrm{AB}$. The emotional distractor images, although irrelevant, could capture attention automatically, thereby gaining preferential access to WM consolidation while blocking the closely following relevant target from gaining similar access. In other words, the irrelevant, negative distractor picture should elicit an N2, reflecting its involuntary attentional selection, followed by a P3b reflecting its consolidation into WM. At short lags, when awareness of the target is suppressed by the preceding negative picture, the amplitudes of these same components that are elicited by the target should be reduced. Furthermore, both the $\mathrm{N} 2$ and P3b components elicited by the negative distractor should be larger on trials associated with errors in reporting the target, as compared with correct trials. These results would provide an explanation for EIB in terms of involuntary capture of the same limited-capacity bottleneck that is implicated in studies of $\mathrm{AB}$.

In summary, if EIB reflects the same trade-off in access to a limited-capacity bottleneck as that observed in studies of the $\mathrm{AB}$, we should find that the irrelevant emotional distractor in the EIB paradigm elicits a $\mathrm{P} 3 \mathrm{~b}$ component and that it is larger on error trials than on correct trials. A similar pattern of results should be observed for the $\mathrm{N} 2$ component, since it appears to be a reflection of an earlier stage in this same limited-capacity bottleneck. The present study examined this prediction.

\section{Method}

Participants

Twenty-six participants ( 9 women, 17 men; mean age, 21.8 years; age range, 19-27) were recruited through a classified ad and were compensated at a rate of $\$ 10$ per hour. Six participants were eliminated for the following reasons. One participant had overall performance accuracy near chance in all conditions. One participant did not show EIB, performing above $90 \%$ accuracy in all conditions. Four participants were eliminated for having recordings with greater than $20 \%$ of segments rejected, due to a combination of noisy channels, blinks, or eye movements. The results are based on the remaining 20 participants. All participants were right-handed and reported normal or corrected-to-normal vision. Each participant provided informed consent, and the experiments were 
approved by the University of Delaware Human Subjects Review Board.

\section{Materials and procedure}

Participants completed the experiment in an electrically shielded room. They viewed stimuli on a 17 -in. Dell CRT monitor $(1,024 \times 768$ pixel resolution) with a refresh rate of $100 \mathrm{~Hz}$. Stimuli were $320 \times 240$ pixel color photographs presented on a gray background. Each picture subtended $8.3^{\circ} \times 6.5^{\circ}$ of visual angle at a viewing distance of $70 \mathrm{~cm}$ (participants used a chinrest). Stimulus presentation was controlled by software written in MATLAB with the Psychophysics Toolbox extensions (Brainard, 1997; Pelli, 1997).

The experiment consisted of 650 trials divided into five blocks. On each trial, participants viewed a sequence of 17 color pictures, with each image replacing the previous one every $100 \mathrm{~ms}$. Pictures appeared in the center of the screen against a gray background. The participants' task was to search for a single target, which was a rotated image $\left(90^{\circ}\right.$ left or right) among a series of upright images (see Fig. 1). Fifty trials contained no target or distractor and were not included in the analyses; the remaining 600 trials were divided evenly in a 2 ("lag") $\times 3$ (distractor type) design. The target was presented either two (lag 2) or eight (lag 8) positions after a distractor picture, which randomly appeared as the fourth or sixth picture in the stream. Distractor pictures were one of three types: emotionally arousing negative, neutral, or "baseline" (another scene picture randomly selected from the same set as the "filler" items in the stream). Participants indicated the direction in which the target picture was rotated (left or right) and
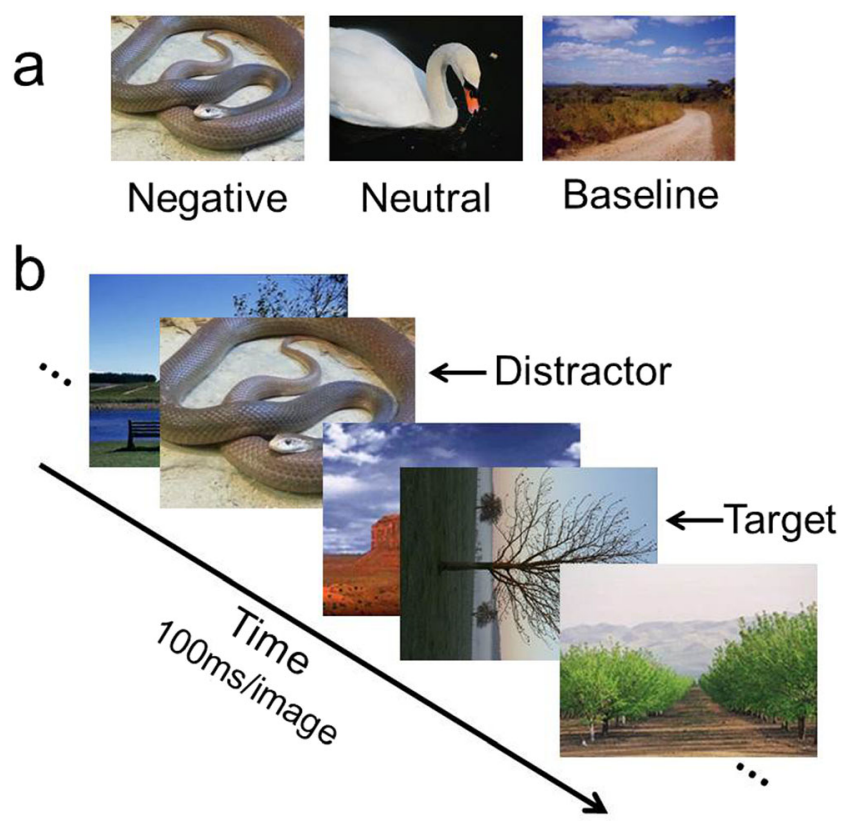

Fig. 1 a Types of distractors and $\mathbf{b}$ schematic design of the study. See the text for details rated their confidence on a 3-point scale (very sure, somewhat sure, or guess).

One hundred twenty-eight images served as targets (64 landscape and architectural images rotated $90^{\circ}$ both clockwise and counterclockwise). Filler images came from a bank of 252 upright landscape and architectural photographs. There were 56 negative distractor pictures (depictions of medical trauma, threatening animals, and violence) and 56 neutral distractor pictures (depictions of people or animals with no obvious emotional content). In the baseline condition, the item occupying what would have been the serial position of the distractor was drawn from the same bank of landscape/ architectural images as the filler items. Negative and neutral images were primarily drawn from the International Affective Picture System (Lang, Bradley, \& Cuthbert, 2001) on the basis of ratings of valence and arousal and were supplemented with similar images from publicly available sources.

At the start of each trial, participants viewed a fixation cross in the center of the screen and clicked the mouse when they were ready to view the stream of stimuli. They were instructed not to move or blink their eyes during the presentation of the images. After the stream of stimuli, a blank screen appeared for $1 \mathrm{~s}$, followed by a response screen depicting six buttons. The six buttons were arranged into two groups of three, which corresponded to rotation direction (left vs. right) and confidence (very sure, somewhat sure, or guess). Categorization of response accuracy incorporated the confidence judgments as a way to eliminate correct guesses. Responses were categorized as correct if (1) they matched the orientation of the target picture and (2) the corresponding confidence rating was either very sure or somewhat sure. Responses were categorized as incorrect if participants reported the wrong orientation or reported that they were guessing.

Before starting the experiment, participants were explicitly told that images of people and animals would appear in the experiment, that they would sometimes be unpleasant, and that they would never be the rotated target. They were also shown examples of emotional and neutral images to ensure that they were comfortable completing the experiment and were reminded several times that they could withdraw from the experiment at any time. They then engaged in a short 16trial practice session, with RSVP rates starting at 5 images per second and increasing to the experiment presentation rate of 10 images per second. The practice session did not include distractors. Participants were debriefed at the end of the experiment.

Electrophysiological recording and data analysis

The electroencephalogram (EEG) was recorded with an Electrical Geodesics Inc. system (EGI; Eugene, OR) using a 129channel Hydrocel Sensor Net. Individual electrode impedances were kept below $50-75 \mathrm{k} \Omega$, as recommended by the 
manufacturer. The data were referenced online to the vertex, band-pass filtered from 0.01 to $80 \mathrm{~Hz}$, and digitized at $200 \mathrm{~Hz}$. Subsequent processing was performed offline using EGI Net Station 4.1.2 software. The data were low-pass filtered with a cutoff of $40 \mathrm{~Hz}$ and then segmented using an epoch that began $200 \mathrm{~ms}$ prior to the onset of the distractor picture and ended 1,200 ms after onset. Channels were marked as bad if the maximum voltage range exceeded $100 \mu \mathrm{V}$. Individual segments were rejected if more than 10 channels were marked as bad. Trials containing blinks (threshold $=100 \mu \mathrm{V}$ ) or eye movements (threshold $=70 \mu \mathrm{V}$ ) were rejected. For the remaining segments, bad channels were replaced by interpolating from surrounding channels. Finally, the segments were averaged, rereferenced to the average reference, and baseline corrected using the 200-ms prestimulus interval.

\section{Data analysis procedures}

Difference waves were used to isolate ERP components elicited by distractor and target pictures from the periodic ERP activity generated by the sequence of pictures (see Vogel et al., 1998). This is particularly important in the present experiment, which used a rapid sequence of pictures. Each background picture produces its own set of sensory components every $100 \mathrm{~ms}$, and effects of the various critical events, such as the presentation of the distractor and target pictures, produce their own ERPs superimposed on the background activity. As Vogel et al. (1998) demonstrated, subtracting the ERP in one condition from another can isolate the ERPs associated with the critical events. For example, ERP components elicited by the negative distractor picture can be isolated by subtracting the baseline lag 8 condition from the negative lag 8 condition. In the first $1,000 \mathrm{~ms}$ of the recording epoch, these conditions differ only in the presence or absence of the distractor, so the subtraction will isolate the ERP components due to the presence of a distractor. Similarly, components elicited by the target picture can be isolated by subtracting the lag 8 condition from the corresponding lag 2 condition for each type of distractor. For example, the components elicited by the target picture when it is preceded by a negative distractor can be isolated by subtracting the negative lag 8 condition from the negative lag 2 condition. Both conditions have a negative distractor in common, so the associated components will subtract out. The two conditions differ only in the presence or absence of a target, so target components will be isolated in this subtraction.

The sensor locations corresponding to the two components of interest in this study (the N2/EPN and the P3b) were chosen using a method recommended by Keil et al. (2014) to minimize the inflation of type 1 error that would accompany choosing sensors on the basis of the largest signal observed in the entire set of sensors. We first averaged over the three distractor conditions separately for the lag 2 and lag 8 conditions. We then computed the lag2 - lag8 subtraction, which should reveal the P3b and N2 components elicited by the target. A second subtraction used the average across distractor conditions at lag 8 but subtracted the baseline lag 8 curve to isolate the $\mathrm{N} 2$ elicited by the distractors. As is detailed below, we also observed a positivity following the negative and neutral distractor pictures that had the same scalp distribution as the $\mathrm{N} 2$ so we used these sensors to measure its amplitude. Clusters of three to six spatially contiguous sensors that showed the maximum amplitude in the relevant difference wave were averaged together. These difference curves were also used to specify time windows for measuring average amplitude. The beginning and end points of the averaging window were chosen corresponding to the points at which the amplitude was half of the peak value (Picton et al., 2000).

The sensors that we chose for the N2/EPN were centered on $\mathrm{PO} 7$ and PO8 in the left and right hemispheres, respectively, while the $\mathrm{P} 3 \mathrm{~b}$ was centered on a location midway between $\mathrm{Pz}$ and CPz. These locations are in good agreement with previous research on these two components (e.g., Schupp, Junghöfer, Weike, \& Hamm, 2003a). Amplitude measures were analyzed with a repeated measures analysis of variance (ANOVA), which employed Greenhouse-Geisser corrections for violations of sphericity. Significant main effects involving three levels of a factor (e.g., negative, neutral, and baseline distractors) were followed up with least significant difference tests between each pair of means. This procedure does not involve a correction to the alpha level for multiple comparisons, because there is no inflation of family-wise error rates for the special case of three conditions, as long as post hoc tests are preceded by a significant main effect (Cardinal \& Aitken, 2006).

As is described in the Results section, determining whether the distractor picture produced a P3b component was complicated by the finding that the negative and neutral distractor pictures elicited a prominent positivity over posterior sensors in a time window similar to what one would expect for a $\mathrm{P} 3 \mathrm{~b}$ to the distractor picture. This posterior positivity had a scalp distribution that was different from the typical topography of the P3b component, but it appeared to partially overlap with sensors in the P3b region. In order to objectively separate this component from the $\mathrm{P} 3 \mathrm{~b}$, we analyzed the data using a twostep principal components analysis (PCA) that employed the ERP PCA (EP) Toolkit version 2.34 for MATLAB (Dien, 2010). Analyses were based on the covariance matrix with Kaiser normalization. Promax rotations were used for both temporal and spatial steps, thus allowing factors to be correlated. The "parallels" test (Dien, 2010) was used to determine the number of factors to be retained in each step. The spatial step was performed first in order to capitalize on the prominent $\mathrm{P} 3 \mathrm{~b}$ component elicited by targets at lags 2 which should yield a clear spatial P3b factor. A subsequent temporal PCA was conducted using the spatial factors from the first step as 
"virtual electrodes" (Spencer, Dien, \& Donchin, 2001). The temporal factors for the P3b spatial factor should include a time window for the target. Of primary interest was whether we would also obtain an earlier time window corresponding to a P3b elicited by the emotional distractor.

In the first step, a spatial PCA was performed on data from the 129 sensors using average waveforms for each subject consisting of the three distractor type (negative, neutral, and baseline) at lag 2. Each waveform included 280 time points covering the interval from $200 \mathrm{~ms}$ before the appearance of the distractor to $1,200 \mathrm{~ms}$ following it. On the basis of the parallels test (Dien, 2010), we retained 10 spatial factors. In the second step, a temporal PCA was performed on the time points using the combinations of distractor type (negative, neutral, and baseline) $\times 10$ "virtual spatial electrodes" (the spatial factors derived in the first step). Eight temporal factors were retained, yielding 80 factor combinations (10 spatial $\times 8$ temporal factors). We inspected this set of factors to locate the P3b spatial component, which was defined as having a midline positive maximum over posterior scalp locations (centered on $\mathrm{Pz}$ ) and having a large amplitude in response to targets in the baseline condition at lag 2. This approach resulted in a clear P3b spatial factor, allowing us to examine the eight temporal windows to determine whether a P3b was elicited by the distractor pictures.

\section{Results}

Behavioral results

Figure 2 shows percent accuracy (corrected for guessing; see the Method section) in discriminating the orientation of the target when it was preceded by different distractor types (negative, neutral, and baseline) at two different lags. A twofactor ANOVA (distractor type: negative, neutral, baseline $\times$ lag 2 vs. 8) revealed significant effects of distractor type, $F(2$, $38)=72.37, p<.001$, and lag, $F(1,19)=133.69, p<.001$, and their interaction, $F(2,38)=87.73, p<.001$. A separate

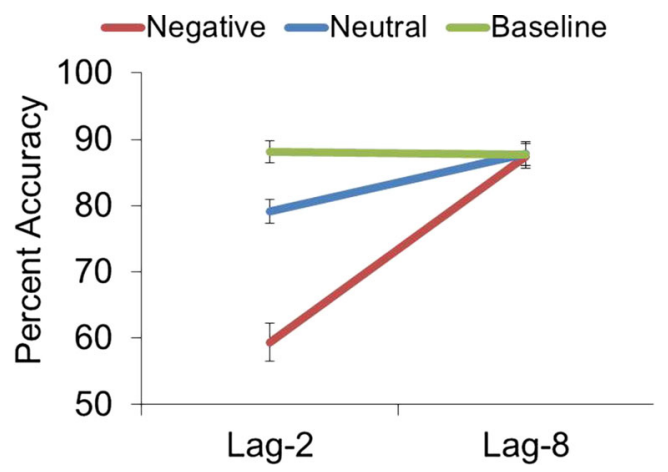

Fig. 2 Behavioral results. See the text for details analysis of the lag 2 data revealed a significant effect of valence, $F(2,38)=108.84, p<.001$. Post hoc tests showed that all pairs of distractor types were significantly different from each other (all $p \mathrm{~s}<.001$ ). This pattern of results is similar to that observed in previous studies of EIB (e.g., Most et al., 2005).

\section{ERP results}

\section{N2}

The N2 component elicited by the distractor pictures was isolated by subtracting the lag 8 baseline condition from the negative and neutral lag 8 conditions. In Fig. 3, the resulting subtraction ERPs are shown in the form of a topographic map (negative distractor, latency $=225 \mathrm{~ms}$ ) and waveforms. The topographic map shows that the distractor N2 is broadly distributed over posterior areas in the left and right hemispheres, with a slight maximum amplitude over the right hemisphere. This topography is consistent with previous reports of the $\mathrm{N} 2$ component to emotional stimuli (the N2 elicited by emotional pictures is often referred to as an early posterior negativity, or EPN; e.g., Schupp et al., 2003a). The $\mathrm{N} 2$ peaks $225 \mathrm{~ms}$ after the onset of the distractor picture and is larger for the negative than for the neutral distractor. We quantified this effect by averaging over three contiguous sensors in each hemisphere in the vicinity of T5 and T6 (64, 68 , and 69 in the left hemisphere; 89, 94, and 95, in the right). The time window (185-275 ms after distractor onset) corresponded to the half amplitude points on either side of the peak (Picton et al., 2000). Although average voltage in the window is the preferred measure of component amplitude, it would produce a distorted value for the neutral distractor N2 as it is of shorter duration than the negative distractor N2 and reverses polarity before the end of the measurement window. We circumvented this problem by using the peak negativity in the window as an amplitude measure for this comparison only. A two-factor ANOVA (hemisphere $\times$ distractor type: negative and neutral) on the peak values revealed that the $\mathrm{N} 2$ was larger for a negative distractor than for a neutral one, $F(1,19)=$ $28.28, p<.001$. Neither the main effect of hemisphere, $F(1$, $19)=2.47, p=.10$, nor its interaction with distractor type, $F<$ 1 , was significant. In addition, single-sample $t$-tests showed that the peak amplitude was significantly different from zero for both the negative distractor, $t(19)=7.24, p<.001$, and neutral distractor, $t(19)=2.90, p=.009$, indicating that both $\mathrm{N} 2 \mathrm{~s}$ were larger than the N2 observed in the baseline condition.

The N2 component elicited by the target was isolated by subtracting the corresponding lag 8 condition from each lag 2 condition. For example, the target $\mathrm{N} 2$ in the negative distractor condition was obtained by subtracting the negative lag 8 condition from the negative lag 2 condition. This subtraction isolates the target effect, because the only difference between 
Fig. 3 Distractor N2 topography and waveforms. The topographic map displays the negative minus baseline distractor conditions when the target appeared at lag 8 (at the peak latency of $225 \mathrm{~ms}$ post-distractor-onset). Waveforms were averaged across contiguous electrode sites (represented in white) and show the negativebaseline and neutral-baseline difference curves when the target appeared at lag 8 . The gray rectangle shows the measurement window (185-275 ms postdistractor-onset)

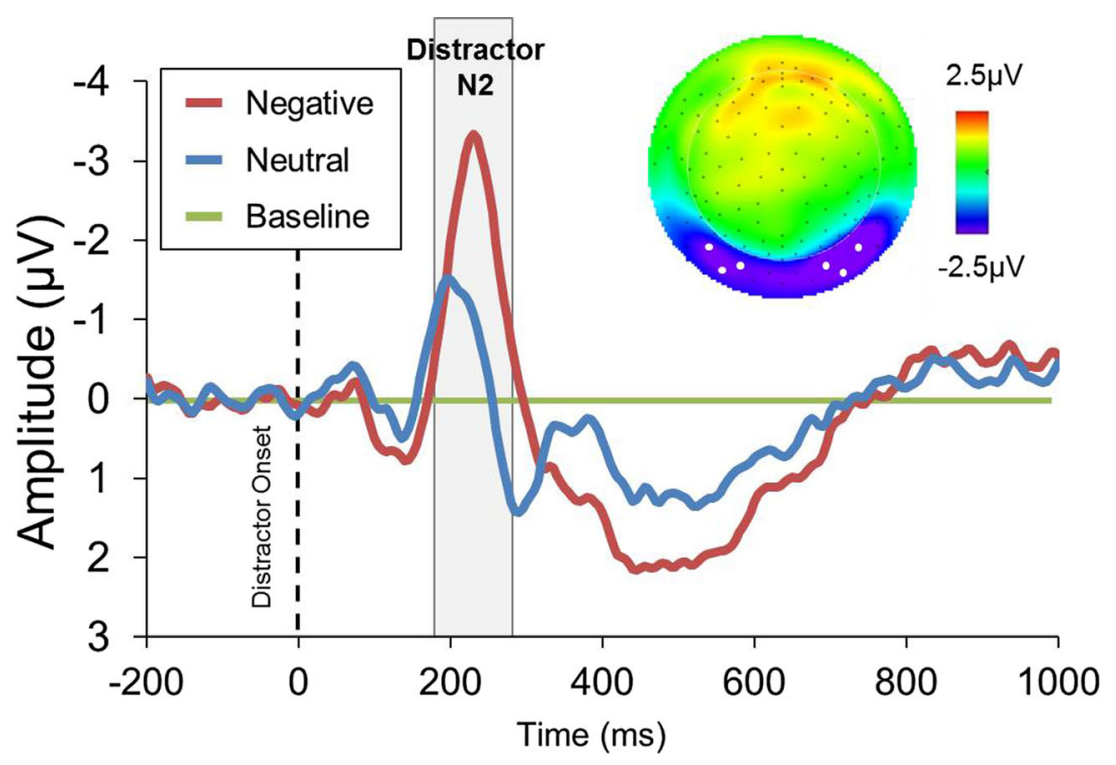

these conditions in the first $800 \mathrm{~ms}$ postdistractor is the presence of a target picture in the lag 2 condition. A similar subtraction was used to isolate the target N2 in the neutral condition (lag 2 neutral minus lag 8 neutral) and in the baseline condition (lag 2 baseline minus lag 8 baseline).

Figure 4 shows the resulting topographic maps and waveforms. The scalp distribution of the target $\mathrm{N} 2$ is similar to that of the distractor N2, with a broad posterior distribution and a slight maximum over the right hemisphere. The peak negativity occurred at $250 \mathrm{~ms}$ following the onset of the target. The amplitude of the target N2 was largest when it was preceded by a baseline distractor and smallest when it was preceded by a negative distractor. Amplitude was intermediate in the case of a neutral distractor. To quantify these effects, we used the same six sensors (three in each hemisphere) used above for the distractor N2 and a time window of 205-385 ms following target onset. An ANOVA using the factors of hemisphere and distractor type showed no main effect of hemisphere, $F<1$, and a significant main effect of distractor type, $F(2,38)=9.51$, $p<.001$. The interaction was not significant, $F<1$. Post hoc paired comparisons revealed that the target N2 was significantly larger when the target was preceded by a baseline distractor, as compared with either a negative $(p<.001)$ or a neutral $(p=.04)$ distractor. The negative and neutral distractor conditions were also significantly different $(p=.04)$.

\section{Target P3b}

The P3b component elicited by the target picture at lag 2 for each condition was isolated by subtracting the lag 8 waveform from its corresponding lag 2 waveform for each of the three distractor conditions. These waveforms are shown in Fig. 5. On the basis of a combination of prior research (e.g., Polich, 2012), a PCA we conducted (see below), and the observed scalp topography, we quantified the $\mathrm{P} 3 \mathrm{~b}$ as the average amplitude for sensors $54,55,79,78,61$, and 62 , which is located between $\mathrm{Cz}$ and $\mathrm{Pz}$ in the 10-20 system (white dots in Fig. 5). The measurement window extended from 435 to $795 \mathrm{~ms}$ relative to target onset (635-995 ms relative to distractor onset). A oneway repeated measures ANOVA revealed a main effect of distractor type, $F(2,38)=16.11, p<.001$. Pairwise comparisons showed that the negative distractor condition produced a

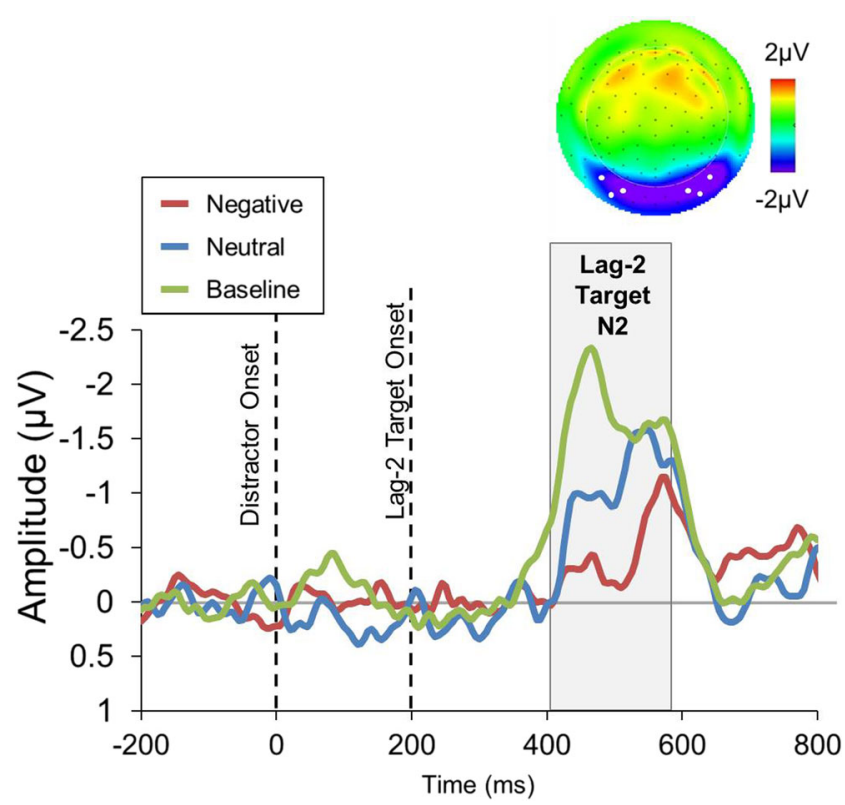

Fig. 4 N2 topography and waveforms elicited by the target picture appearing at $200 \mathrm{~ms}$. The topographic map displays the negative minus baseline lag 2 conditions (at the peak latency of $250 \mathrm{~ms}$ post-target-onset). Waveforms were averaged across contiguous electrode sites (represented in white) and show the negative lag 2 - negative lag 8 , neutral lag $2-$ neutral lag 8 , and baseline lag 2 - baseline lag 8 subtraction waveforms. The gray rectangle shows the measurement window (205-385 ms posttarget-onset) 
Fig. 5 Scale topographies and difference curves for the $\mathrm{P} 3 \mathrm{~b}$ to the target picture appearing at $200 \mathrm{~ms}$. The topographic map displays the negative minus baseline lag 2 conditions at the latency of $520 \mathrm{~ms}$ post-targetonset ( $720 \mathrm{~ms}$ post-distractoronset). Waveforms were averaged across contiguous electrode sites (represented in white) and show the negative lag 2 - negative lag 8 , neutral lag $2-$ neutral lag 8 , and baseline lag 2 - baseline lag 8 subtraction waveforms. The gray rectangle shows the measurement window (435-795 ms post-targetonset)

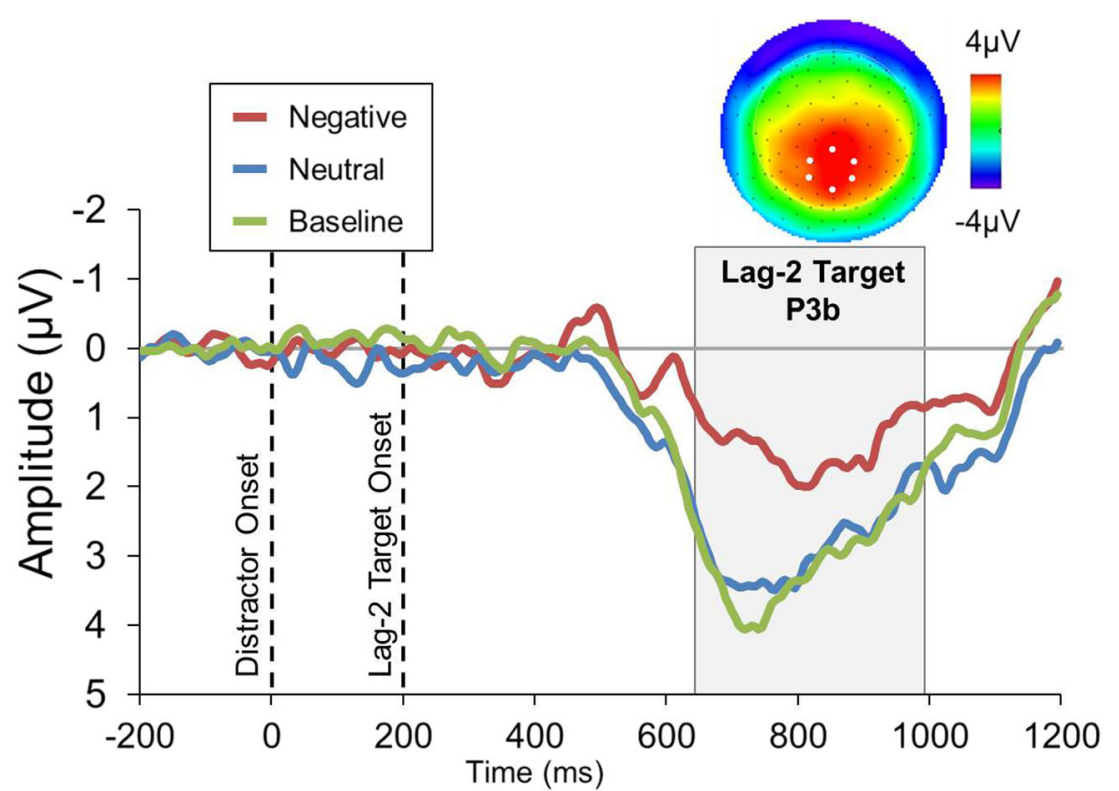

smaller target P3b than did both the neutral $(p<.001)$ and baseline $(p<.001)$ conditions. The neutral and baseline conditions were not significantly different $(p=.42)$. These results are generally consistent with the behavioral accuracy data, which showed a large blink for the negative distractor and a much smaller one for the neutral condition. It might be that the difference in accuracy between the neutral and baseline conditions was simply too small to reliably affect $\mathrm{P} 3 \mathrm{~b}$ amplitude.

\section{Distractor $P 3 b$}

In order to determine whether the distractor pictures elicited a P3b, we subtracted the lag 8 baseline condition from the lag 8 negative and lag 8 neutral conditions. The resulting subtraction curves are shown in Fig. 6. The waveforms represent the average of the same six sensors that were used to quantify the target $\mathrm{P} 3 \mathrm{~b}$. For illustration purposes, this figure also contains the waveform for the target $\mathrm{P} 3 \mathrm{~b}$ elicited in the baseline condition (lag 2 baseline minus lag 8 baseline), which did not have a P3b in the time interval corresponding to the distractor. To quantify P3b amplitude, we used a measurement window extending from 400 to $550 \mathrm{~ms}$ following distractor onset. A one-way repeated measures ANOVA revealed a significant effect of distractor type, $F(2,38)=14.36, p<$ .001. Pairwise comparisons showed that the negative distractor condition produced a larger $\mathrm{P} 3 \mathrm{~b}$ than did both the neutral $(p<.001)$ and baseline $(p=.012)$ conditions. The neutral and baseline conditions were not significantly different
Fig. 6 Distractor P3b topographies and difference curves. The topographic map displays the negative minus baseline lag 8 conditions (at the latency of $485 \mathrm{~ms}$ post-distractoronset). The negative and neutral waveforms represent distractors with no target presented until lag 8 (using the negative-baseline and neutral-baseline difference waves). The baseline condition serves as a comparison and shows the P3b for lag 2 targets that appear after baseline distractors (lag 2 baseline - lag 8 baseline difference wave). The gray rectangle shows the measurement window (400-550 ms postdistractor-onset). See the text for details

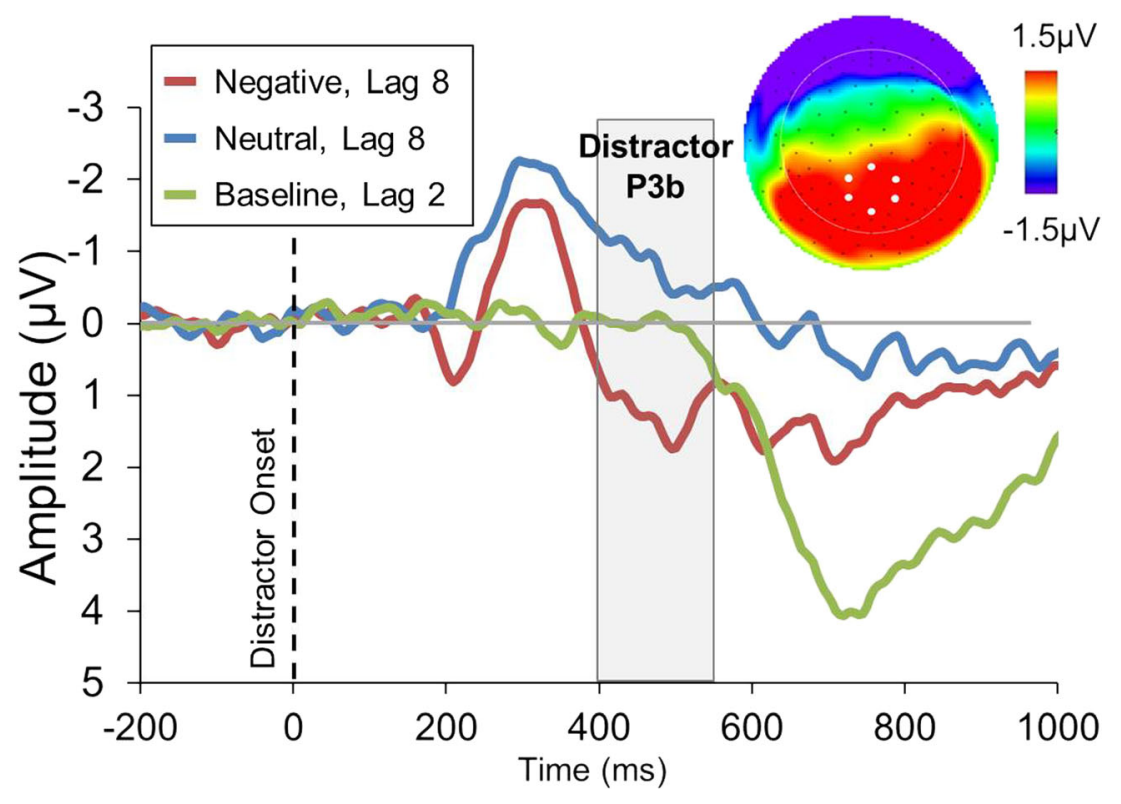




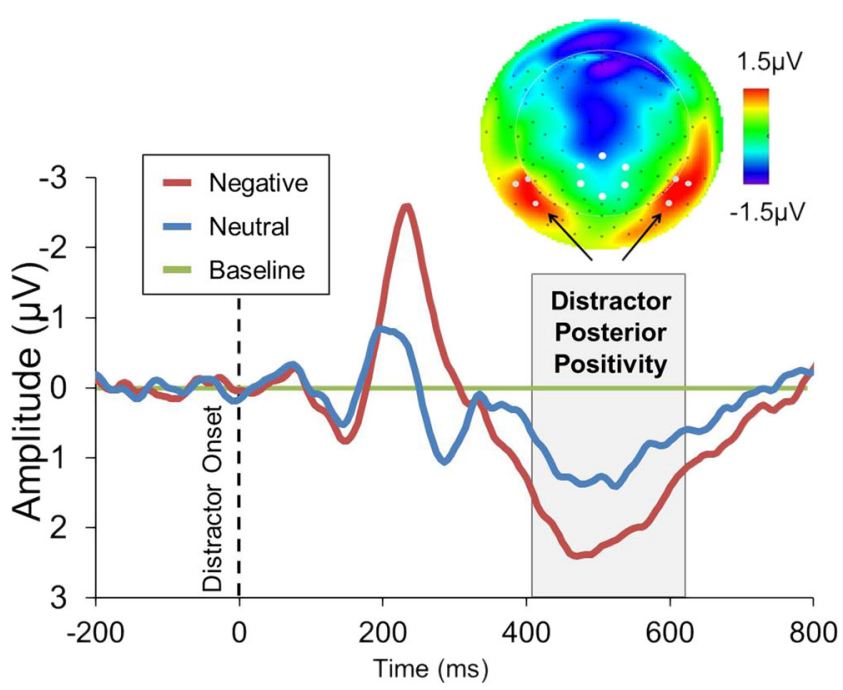

Fig. 7 Topographic map and waveforms for the posterior positivity component. The topographic map displays the neutral minus baseline distractor conditions (at the peak latency of $470 \mathrm{~ms}$ post-distractor-onset) in the lag 8 condition. Waveforms were averaged across contiguous electrode sites (represented in white and indicated with arrows) and show the negative - baseline and neutral - baseline difference curves when the target appeared at lag 8. Electrode sites for the P3b are also shown for reference. Waveform data have been averaged over hemispheres. The gray rectangle shows the measurement window (265-465 ms postdistractor-onset)

$(p=.053)$, and no $\mathrm{P} 3 \mathrm{~b}$ was apparent for the neutral distractor. We revisit this issue in the PCA section below.

\section{Posterior positivity}

The N2 to the distractor was followed by a positivity with a similar scalp distribution, shown in Fig. 7 for the negative and neutral distractor conditions. This component was isolated by subtracting the lag 8 baseline condition from the lag 8 negative and lag 8 neutral conditions. None of these contained a target in the first $800 \mathrm{~ms}$ of the recording epoch, so any resulting activity should be attributable to the distractor pictures. The subtraction topography shows a bilateral, posterior positivity over occipital temporal areas that is slightly larger over the right hemisphere than over the left. We will refer to this as the posterior positivity component, although we speculate in the discussion that it might be identical to the $\mathrm{P}_{\mathrm{D}}$ component that is elicited by salient, task-irrelevant singletons in visual search tasks (Kiss, Grubert, Petersen, \& Eimer, 2012; Sawaki et al., 2012; Sawaki \& Luck, 2010). We quantified the average amplitude of this component using a measurement window of 405-615 ms after distractor onset. The sensors used to assess its amplitude were 96,100 , and 101 in the right hemisphere (close to $\mathrm{T} 6$ and $\mathrm{O} 2$ in the 10-20 system) and 64, 68, and 69 in the left hemisphere. Notably, these sensors are similar to those used to measure the earlier N2 component, and the peak latency of this component $(470 \mathrm{~ms})$ is close to the latency of the $\mathrm{N} 2$ to lag 2 baseline targets $(265 \mathrm{~ms}$ posttarget and $465 \mathrm{~ms}$ postdistractor). This component can also be seen in the topographic map in Fig. 6, where it overlaps with the P3b elicited by the negative distractor. An ANOVA revealed that the posterior positivity was larger for negative than for neutral distractors, $F(1,19)=16.68, p=.001$. There was no main effect of hemisphere, $F(1,19)=1.31, p=.268$, or of the interaction of hemisphere and distractor type, $F(1,19)=4.19$, $p=.055$. However, the partial overlap in time and sensor locations between the posterior positivity and the P $3 \mathrm{~b}$ elicited by the negative distractor makes it difficult to know how much our measurement of the posterior positivity is confounded by effects of the distractor P $3 b$. We addressed these issues by separating the components using a PCA.

Principal components analysis

In order to separate the P3b components elicited by the negative distractor and the following target at lag 2 and to ensure that the posterior positivity is a separate component from the distractor P3b, we conducted a two-step, spatial-temporal PCA (Dien, 2010; see the Method section) on the 1,200-ms interval following onset of the distractor picture.

The first step was a spatial PCA. This should isolate a spatial $\mathrm{P} 3 \mathrm{~b}$ component due to the presence of a robust $\mathrm{P} 3 \mathrm{~b}$ to the lag 2 target in the baseline condition. Applying a temporal PCA to this spatial factor should produce two time windows corresponding to P3bs elicited by the distractor and the lag 2 target. We retained 10 spatial factors from the first step and 8 temporal factors from the second step (see the Method section for details). Figure 8 shows the scalp topography for spatial factor 2, which corresponds well with the known distribution of the centro-parietal P3b. It is also consistent with the topography of the target P3b shown in Fig. 5 . Temporal factors one and three (S2T1 and S2T3) for this spatial component peak at $575 \mathrm{~ms}$ following the target and $485 \mathrm{~ms}$ following the distractor. Figure 8 plots these factors for the baseline lag 2 and negative lag 8 conditions, respectively. In order to facilitate comparison with the earlier peak analysis, we quantified the PCA for the lag 2 target (S2T1) in terms of the difference between lags 2 and 8.These difference scores were $2.01,4.03$, and $4.42 \mu \mathrm{V}$ for negative, neutral, and baseline conditions, respectively. An ANOVA on these data revealed a significant effect of distractor type, $F(2,38)=$ $22.17, p<.001$. Post hoc comparisons showed that the negative distractor condition resulted in a smaller target $\mathrm{P} 3 \mathrm{~b}$ than did both the neutral $(p<.001)$ and baseline conditions $(p<$ $.001)$, which did not differ $(p=.28)$. These results are in agreement with the results of the corresponding peak analysis presented earlier in showing that the $\mathrm{P} 3 \mathrm{~b}$ to the target was suppressed following a negative distractor.

A similar analysis was conducted on factor scores for S2T3, which appears to be a P3b elicited by the distractor picture. A two-factor (distractor type $\times$ lag) ANOVA 
Fig. 8 P3b topography for spatial factor 2 from the PCA. The topographic map displays at the latency of $575 \mathrm{~ms}$ post-targetonset. The two waveforms depicted represent the $\mathrm{P} 3 \mathrm{~b}$ elicited by the negative distractor (lag 8 ) and a target presented at lag 2 after a baseline distractor

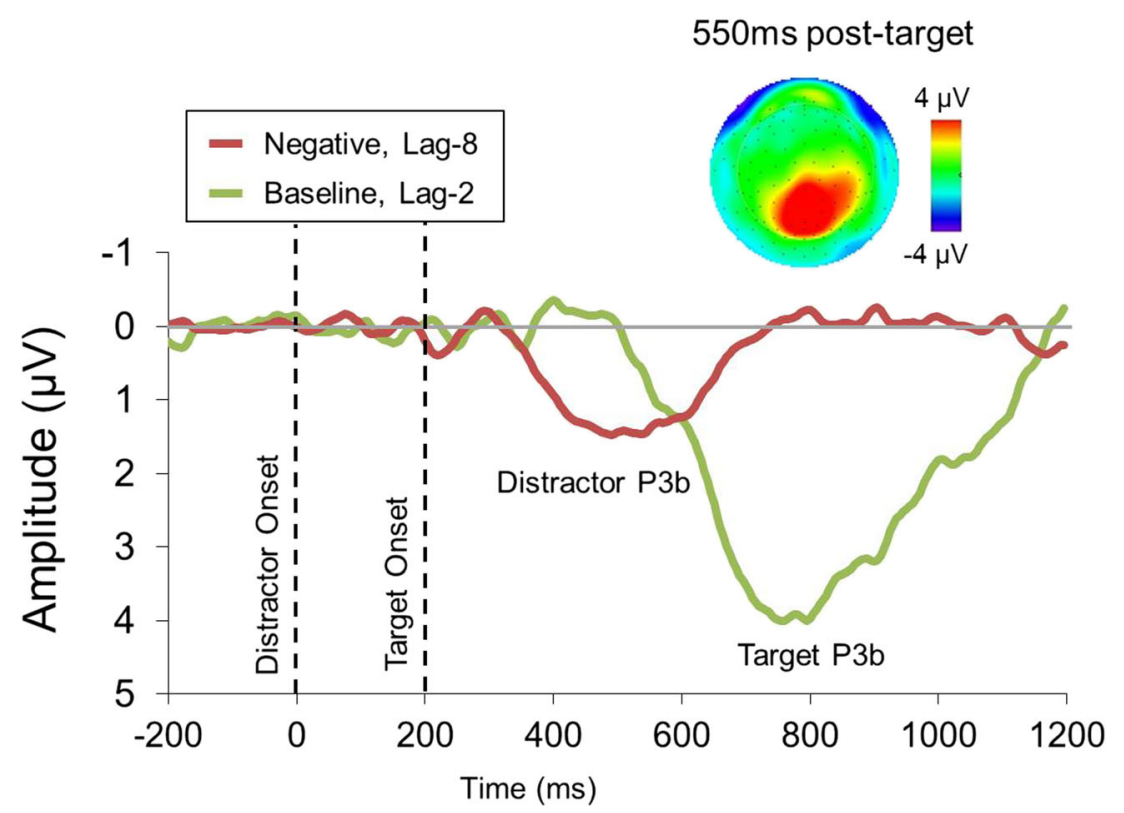

conducted on the factor scores revealed no main effect or interaction involving lag $(F<1$ and $F(2,38)=1.16, p=.325$, respectively). There was a main effect of distractor type, with means of $1.51 \mathrm{~V},-0.68 \mathrm{~V}$, and $-0.21 \mathrm{~V}$ for the negative, neutral, and baseline conditions, respectively. This resulted in a main effect of distractor type, $F(2,38)=23.15, p<.001$. Post hoc comparisons showed that the distractor $\mathrm{P} 3 \mathrm{~b}$ was larger in the negative condition than both the neutral $(p<.001)$ and the baseline conditions $(p=.001)$. The neutral and baseline conditions were not significantly different from each other $(p=.094)$. These results provide a confirmation of the results obtained with the amplitude measures of the distractor P $3 b$ presented earlier and indicate that the negative distractor elicited a P3b component even though it was irrelevant to the participant's task.

The PCA also revealed factors corresponding to the posterior positivity component. Separate factors were found for the left (S6T2, latency $=465 \mathrm{~ms})$ and right (S7T2, latency $=$ $465 \mathrm{~ms}$ ) hemispheres, and topographic plots of these factors are shown in Fig. 9, along with waveform data for the three distractor types in the lag 8 condition. An ANOVA with the factors of distractor type, hemisphere, and lag showed no significant main effect of hemisphere $(F<1)$ or any of its interactions with other variables (all $p \mathrm{~s}>.15$ ). The waveform data, averaged over hemisphere, show that the posterior positivity was largest for negative distractors, intermediate for neutral distractors, and smallest for the baseline distractors, resulting in a significant distractor effect, $F(2,38)=71.85, p<$ .001. Pairwise comparisons revealed that all three distractor conditions were significantly different from each other (all $p$ s $<.001)$.

The important contribution of the PCA is the confirmation that the negative distractor picture elicited a P3b component that was separate from a more posterior positivity that occurred in the same time window. We discuss the import of these findings in the Discussion section. But first, we explored which of these components were related to accuracy in reporting the target.

\section{Incorrect versus correct trials}

By examining which ERP components were affected by whether participants were correct or not on the target discrimination, one can identify those components that are related to the "blink" produced by the negative pictures. We were

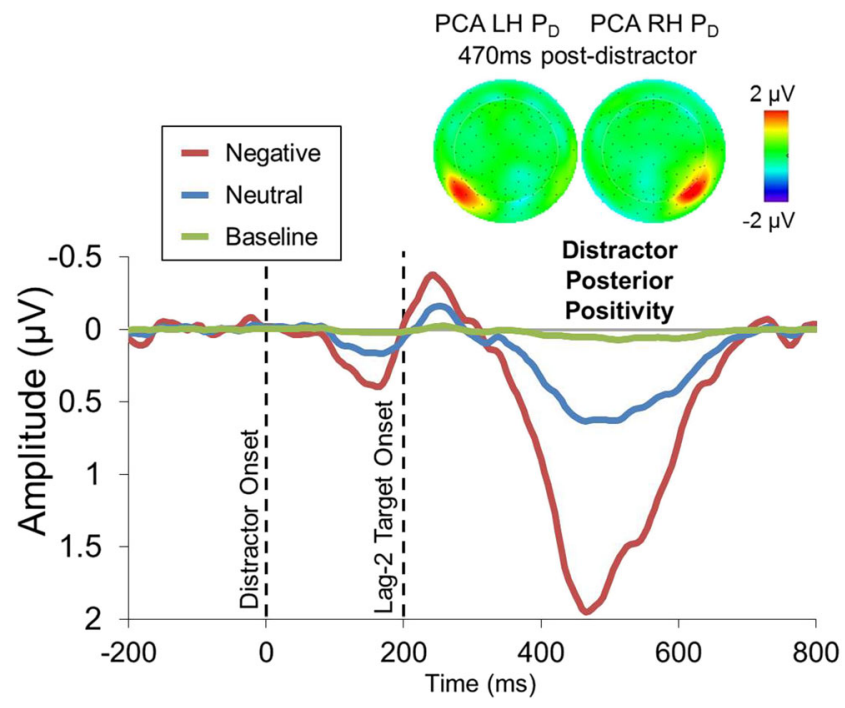

Fig. 9 Posterior positivity topography for spatial factors 6 (left hemisphere, $\mathrm{LH}$ ) and 7 (right hemisphere, $\mathrm{RH}$ ) from the principal component analysis (PCA). Topographic maps display these factors at peak latencies (505 and $465 \mathrm{~ms}$ post-distractor, respectively). Waveforms represent the average of the LH and RH spatial factors at temporal factor 2 in the lag 8 conditions for each distractor type 
Fig. 10 Waveforms for incorrect and correct trials, as well as their difference (incorrect - correct). The topographic maps represent the difference scores at a latency of $290 \mathrm{~ms}$ for the N2 and $535 \mathrm{~ms}$ for the posterior positivity. Waveforms display data from the sensors depicted in white (the same sensors used to measure the $\mathrm{N} 2$ and posterior positivity in earlier analyses)

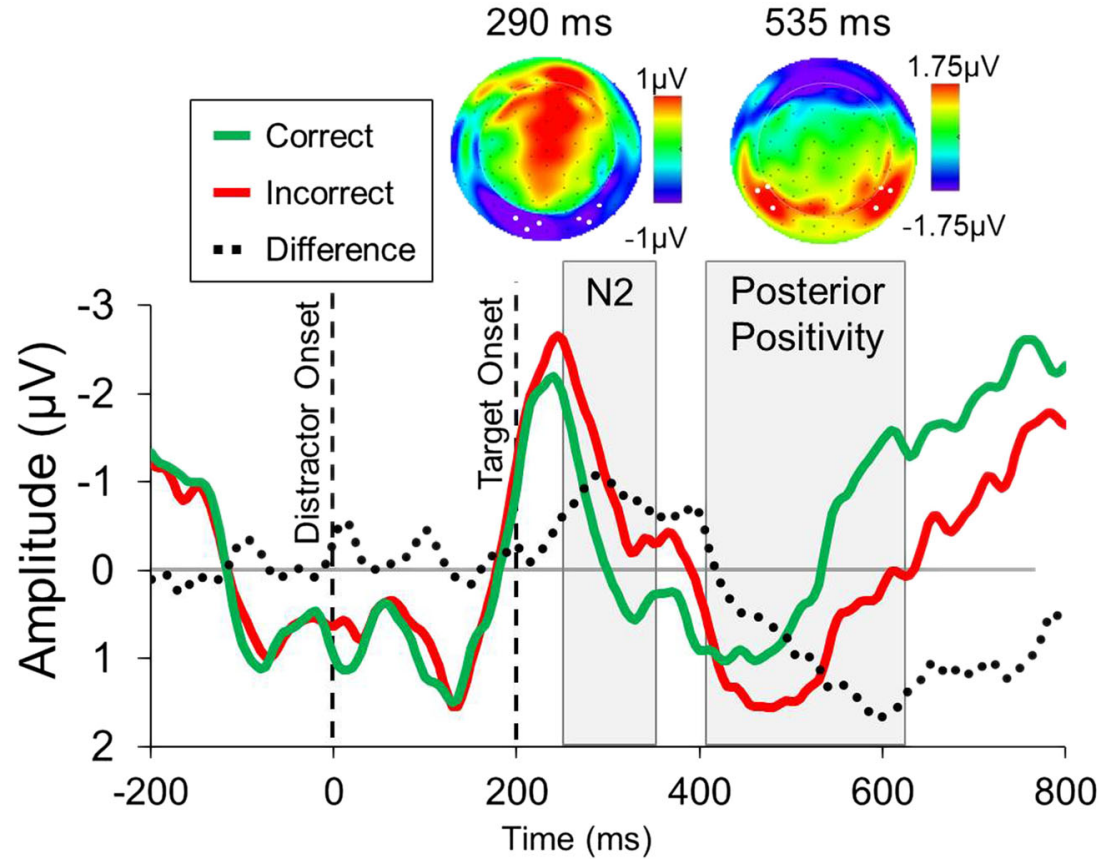

particularly interested in whether any of the three components examined above - the N2, P3b, or posterior positivity-were associated with errors in reporting the target. To evaluate this, we examined ERPs for correct and incorrect trials in the lag 2 negative distractor condition, since this was the only condition in which there were sufficient errors to make this analysis feasible (across participants, the number of error trials in this condition ranged from 21 to 66, with a mean of 42). We used the same sensor clusters and time windows used above to examine the impact of accuracy on the different components.

\section{Distractor N2 and posterior positivity}

Figure 11 shows the N2 topographic map and waveforms for correct and incorrect trials, as well as their difference, which has a bilateral distribution similar to that of the N2 (see Fig. 3); consequently, we used the same sensors to quantify it. The incorrect - correct difference curve peaks at $290 \mathrm{~ms}$, which is later than the peak we found for the distractor N2 $(225 \mathrm{~ms})$ when it was based on the average of correct and incorrect trials. An examination of the separate waveforms for correct and incorrect trials makes it clear that the effect of accuracy is reflected in a longer duration of the distractor $\mathrm{N} 2$ on incorrect trials. We examined the significance of this effect using a window extending from 250 to $350 \mathrm{~ms}$. A $t$-test showed that there was no significant difference between left and right hemispheres, $t(19)=0.818, p=.424$, so we averaged over them. A second $t$-test showed that the average amplitude was greater than zero, $t(19)=3.34, p=.003$. These results show that participants were more likely to make an error on the target when the preceding negative distractor elicited a late or extended $\mathrm{N} 2$.

The initial negativity associated with incorrect trials was followed by a positivity that appears to have the same scalp topography as the posterior positivity examined earlier (see topographic map in Fig. 10). Therefore, we used the same right-hemisphere sensors employed in the earlier analysis and a time window extending from 410 to $650 \mathrm{~ms}$ following the distractor. A $t$-test showed that incorrect trials were associated with a larger distractor posterior positivity than were correct trials, $t(19)=5.12, p<.001$. In addition, it appears that this positivity has an extended time course on error trials.

The prolonged duration of the distractor N2 on incorrect trials may reflect longer engagement of attention on these trials. The subsequent posterior positivity may reflect disengagement of attention, in which case its larger amplitude and extended time course on error trials may reflect greater difficulty in disengaging attention. Therefore, sustained N2 and posterior positivity components elicited by the distractor may index trials on which attention remained engaged on the distractor for a prolonged period, resulting in a failure of the lag 2 target to access the attention system.

\section{Distractor and target P3b}

If errors were at least partly due to the distractor picture occupying WM and preventing access by the target, as appears to be the case in the $\mathrm{AB}$, we would expect larger distractor $\mathrm{P} 3 \mathrm{bs}$ on incorrect trials than on correct trials. Figure 11 shows the topography (at $460 \mathrm{~ms}$ ) and 
waveforms for correct and incorrect trials and their difference. Note that the topographic map for the target $\mathrm{P} 3 \mathrm{~b}$ corresponds to the correct - incorrect difference in order to show a positive P3b. To quantify the P3b, we used the same sensors and time window used above in the section on the Distractor P3b.

The average amplitude in the distractor $\mathrm{P} 3 \mathrm{~b}$ window was $0.29 \mu \mathrm{V}$ for correct trials and $1.00 \mu \mathrm{V}$ for incorrect trials. The difference between these two values was significant, $t(19)=$ $2.57, p=.02$, showing that errors in reporting the target were associated with larger P3bs elicited by the negative distractor picture.

We might also expect that the P3b to targets would be larger on correct trials, relative to incorrect trials, because one could assume that the target was more likely to have gained access to WM on correct trials, and this appears to be the case in the waveforms shown in Fig. 11. A $t$-test on the average amplitude in the interval from 435 to $795 \mathrm{~ms}$, relative to target onset (635-995 ms relative to distractor onset), confirmed that the target P3b was larger on correct trials $(2.66 \mu \mathrm{V})$ than on incorrect trials $(1.87 \mu \mathrm{V}), t(19)=2.74, p=$ .013 .

These data show that, relative to correct trials, incorrect trials are associated with a larger distractor $\mathrm{P} 3 \mathrm{~b}$ and a smaller target $\mathrm{P} 3 \mathrm{~b}$. In other words, there is a trading relationship in the amplitudes of the P3bs elicited by the distractor and target pictures, which supports the hypothesis that competition for a central resource (WM consolidation) is at least part of the reason that irrelevant negative distractor pictures suppress awareness for closely following targets.

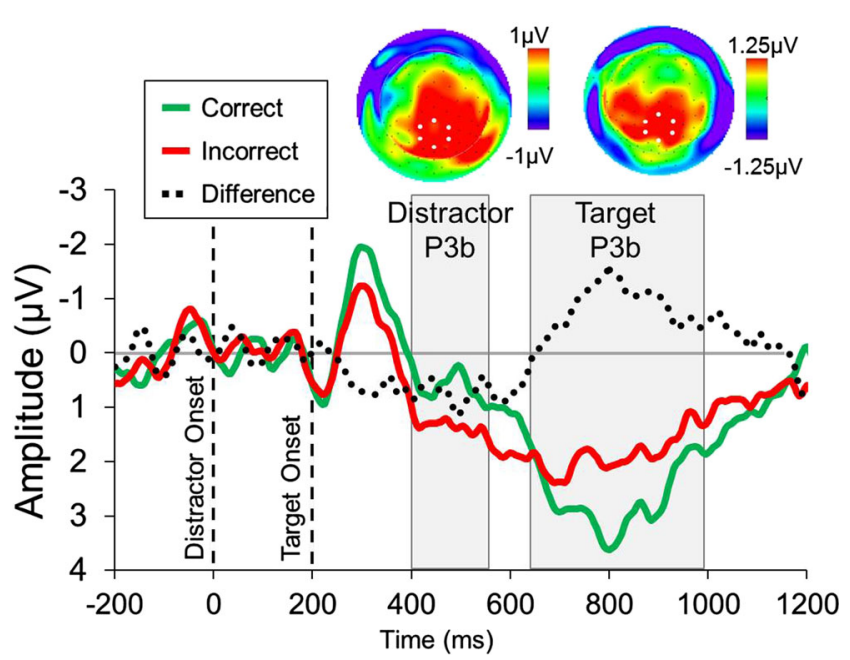

Fig. 11 Waveforms and topographic maps for the incorrect - correct difference using the P3b sensors. Topographic plots display the incorrect - correct difference at a latency of $500 \mathrm{~ms}$ for the distractor and $800 \mathrm{~ms}$ for the target. Waveforms display data from the sensors depicted in white (the same sensors used to measure the P3b in earlier analyses)

\section{Discussion}

The present experiment used ERPs to examine the mechanisms responsible for EIB, the ability of a task-irrelevant, negative picture to suppress awareness of a closely following target picture. Given the phenomenal similarity between EIB and the $A B$, we were specifically interested in whether the processes that are thought to drive the $\mathrm{AB}$ might also be responsible for EIB.

According to the central interference account of the $\mathrm{AB}$, the inability to report the second of two targets presented in close temporal proximity reflects competition at a relatively late bottleneck in target processing, such as consolidation of information into WM (e.g., Chun \& Potter, 1995; for reviews, see Dux \& Marois, 2009; Martens \& Wyble, 2010). In particular, this model assumes that $\mathrm{T} 1$ occupies the consolidation process for an extended period of time, so that a closely following T2 has to queue to gain access and is vulnerable to masking by subsequent distractors. Electrophysiological evidence in support of this account has been provided by experiments that used the P3b component (Kranczioch et al., 2007; Vogel et al., 1998) and the magnetic counterpart to the P300, the M300 (Shapiro et Al., 2006; for simplicity, we will refer to both components as the P3b), as a measure of access to the consolidation process. These experiments have shown that the amplitudes of the $\mathrm{P} 3 \mathrm{~b}$ associated with the two targets in the stream have a trading relationship: A larger amplitude P3b elicited by $\mathrm{T} 1$ is associated with a smaller amplitude $\mathrm{P} 3 \mathrm{~b}$ for T2, and vice versa (Kranczioch et al., 2007; Shapiro et al., 2006). In addition, errors in reporting $T 2$ are associated with larger P3bs to T1 (Shapiro et al., 2006; Vogel et al., 1998). Both of these findings are consistent with the predictions of a central interference account of the $\mathrm{AB}$.

In the present study, we found a similar pattern of results. Negative emotional pictures elicited a P3b component even though they were irrelevant to the observer's task. Importantly, error trials were associated with larger distractor P3b components than were correct trials. These data suggest that emotional pictures sometimes "automatically" gain access to WM consolidation, thereby blocking access by the target picture. Thus, central interference is a mechanism that plays a role in both EIB and $\mathrm{AB}$.

The neutral distractor pictures also impaired detection of the following target, albeit much less than the negative distractors (an approximately $9 \%$ reduction in target accuracy for neutral distractors, as compared with $28 \%$ for negative distractors). The neutral distractors did not appear to elicit a $\mathrm{P} 3 \mathrm{~b}$ and produced a smaller reduction in target $\mathrm{P} 3 \mathrm{~b}$ amplitude, relative to negative distractors. These results show the same trading relationship between temporally proximal P3bs that was observed in the $\mathrm{AB}$ and suggest a similar conclusion: Larger P3bs elicited by distractor pictures reflect greater use of a limited-capacity bottleneck mechanism that is required for 
awareness of closely following targets. Consequently, these targets are less likely to gain admittance to the bottleneck, resulting in smaller target $\mathrm{P} 3 \mathrm{~b}$ components. Therefore, the same limited-capacity mechanism that is reflected in the $\mathrm{P} 3 \mathrm{~b}$ component seems to play a role in target suppression in both $\mathrm{EIB}$ and $\mathrm{AB}$.

In addition, variations in two other components of the $\mathrm{ERP}$ - the N2 and a posterior positivity-were related to the occurrence of EIB. First, we consider the N2. In agreement with earlier reports (Flaisch, Junghöfer, Bradley, Schupp, \& Lang, 2008; Peyk, Schupp, Keil, Elbert, \& Junghöfer, 2009; Schupp, Junghöfer, Weike, \& Hamm, 2003a, 2003b), we found that emotional pictures produced a larger N2 component, as compared with neutral and baseline pictures. The target picture, when it was not preceded by a distractor, also elicited a prominent negative component that had a latency and scalp distribution that was similar to the N2 elicited by distractors. Given the similar temporal and topographic patterns, both target and distractor N2s appear to be produced by the same neural generator, which suggests that the N2 elicited by emotional pictures, known as the EPN in the emotion literature, may not be uniquely associated with emotional stimuli, in agreement with earlier studies (see Schupp, Flaisch, Stockburger, \& Junghöfer, 2006). The significant N2 generated by the neutral distractors suggests the same conclusion.

The N2 to the target picture was largely eliminated when the target was preceded by a distractor picture, and the magnitude of suppression was larger following negative distractors, as compared with neutral distractors. Negative distractors themselves produced a larger N2 than did neutral distractors, and thus the amplitudes of the N2s for distractors and targets exhibited a trading relationship similar to that which was observed for the P3b component: Larger distractor $\mathrm{N} 2 \mathrm{~s}$ were associated with smaller target N2s. Importantly, the distractor N2 was also related to accuracy in reporting the target. Trials on which the observer failed to report the target were associated with temporally extended N2s to the distractor. Thus, the N2 and P3b components appear to act in concert in producing EIB.

The relatively early latency of the $\mathrm{N} 2(\sim 230-250 \mathrm{~ms}$ after stimulus onset) and its scalp distribution over the posterior occipital-temporal cortex are consistent with this component being related to perceptual and/or attentional processes. One possibility is that the $\mathrm{N} 2$ in $\mathrm{AB}$ and EIB paradigms is related to the N2pc component, which is thought to index the allocation of object-based attention (Luck, 2011). Indeed, the N2 observed in the present study is similar in latency and topography to the N2pc. The N2pc is normally strongly lateralized and is measured by subtracting ipsilateral from contralateral activity at posterior electrode sites (Luck, 2011). Our stimuli were presented at fixation, resulting in a bilateral distribution that precluded this subtraction approach. To the degree that these components are the same, we speculate that the N2 reflects the capture of object-based attention by a salient but task-irrelevant picture, as well as by relevant targets. A small target $\mathrm{N} 2$ is associated with a failure of awareness for that target, while a small distractor $\mathrm{N} 2$ reflects its failure to capture attention, thereby resulting in a reduction in EIB. These results suggest that EIB is at least partly due to a competition for attentional resources. When a salient negative distractor picture captures attention, it elicits an N2, and this appears to reflect access to a limited-capacity bottleneck and, ultimately, consolidation into WM reflected in the P3b. In addition, the extended duration of the negative distractor $\mathrm{N} 2$ on error trials may reflect longer attentional engagement times followed by prolonged disengagement, reflected in the posterior positivity component (see below). Sustained attention to the distractor would decrease the likelihood that the target is able to gain access to the selection process required for entry into WM, resulting in a "blink."

Sergent et al. (2005) reported similar findings for the AB task. They found reduced N2 and P3b components elicited by the second target when it was blinked (see also Kranczioch et al., 2007). They noted that at lags producing a blink, the N2 elicited by the second target overlapped in time with the $\mathrm{P} 3 \mathrm{~b}$ elicited by the first target, suggesting that these two components (N2 and $\mathrm{P} 3 \mathrm{~b}$ ) reflect a common limited-capacity process that cannot simultaneously process two temporally proximal target events. They speculated that the N2 is an earlier manifestation of the same limited-capacity process that is reflected in the later P3b. One possibility is that the $\mathrm{N} 2$ component indexes the selection of which objects or events will be passed on to a second process, reflected in the $\mathrm{P} 3 \mathrm{~b}$, which consolidates information into WM. Thus, these two processes, and their associated ERP components, would generally be closely linked. If the selection process reflected by the $\mathrm{N} 2$ is a bottleneck that is limited to one object (or event; see Sheppard, Duncan, Shapiro, \& Hillstrom, 2002) at a time and the duration of this process has an extended time course, it would appear to be a candidate for playing a role in the AB and EIB.

Intriguingly, we also observed a robust posterior positivity following the distractor that was larger for negative than for neutral distractors. This component, which we call the posterior positivity, appeared to persist for a longer time on trials on which the observer failed to report the target, and it had a scalp topography that was similar to that of the $\mathrm{N} 2$ but opposite in polarity. This posterior positivity may be related to the $\mathrm{Pd}$ component (Kiss et al., 2012; Luck \& Hillyard, 1994; Sawaki, Geng, \& Luck, 2012; Sawaki \& Luck, 2010) that is elicited by task-irrelevant distractors in attentional capture paradigms. Such distractors have been found to elicit an N2pc, followed by a Pd, which may reflect a sequence of engagement and disengagement of attention with respect to the distractor object. Like the posterior positivity in our study, the Pd in these recent studies had a scalp topography similar to that of the 
preceding N2pc, leading Sawaki and colleagues to conclude that the "N2pc and Pd reflect related, but opposite, mechanisms" (Sawaki et al., 2012, p. 7). While the posterior positivity component we observed strongly resembles the Pd, we emphasize that inferring that they are the same component is speculative, and future studies need to be conducted to confirm it.

Previous research on attention capture by emotional pictures has concentrated on the EPN/N2, as well as the late positive potential or LPP, which refers to a constellation of positive components that appear to include the $\mathrm{P} 3 \mathrm{~b}$ and a sustained positive slow wave. The LPP is larger for negative than for neutral pictures and also appears to be related to interference associated with irrelevant negative distractors (Weinberg \& Hajcak, 2011). Most previous investigators did not comment on whether they also observed a posterior positivity following negative pictures that overlaps in time with the P3b, but it could have been overlooked because of this overlap. One exception is a study by Foti, Hajack, and Dien (2009), who used temporal-spatial PCA to separate the LPP into the P3b, a positive slow wave, and a posterior positivity that peaked at $343 \mathrm{~ms}$ following the negative picture, with a maximum amplitude at occipital sites.

The scalp topography of this early LPP component appears to be similar to the posterior positivity in the present article, while its earlier onset is probably related to the slower presentation rates used by Foti et al. (2009; their P3b to emotional pictures was also approximately $100 \mathrm{~ms}$ earlier than ours). They interpreted this component as being related to the P3b, while we are suggesting that it might reflect a different underlying process than the $\mathrm{P} 3 \mathrm{~b}$ - namely, disengagement from the emotional picture. Consistent with the latter claim, we found that that the correlation between scores on the two factors in our PCA that corresponded to the P $3 \mathrm{~b}$ and posterior positivity components was not significantly greater than zero, $r=-.008$, $p=.973$, despite our use of a Promax rotation, which allows factors to be correlated. Future research should be directed at exploring experimental manipulations that might dissociate these two components.

\section{Conclusions}

We explored the neural signature of EIB, a phenomenon whereby emotional distractors impair the detection of targets that appear soon after them. We found that negative emotional distractors elicit a large N2 component (perhaps reflecting automatic capture of attention) and a P3b component (thought to reflect consolidation of information into WM). The amplitudes of these components were inversely related to the amplitude of the same components elicited by the target, such that targets presented soon after emotional distractors showed small N2s and small P3bs. In addition, the likelihood of errors in reporting the target in the EIB task was related to the magnitude and timing of the $\mathrm{N} 2$ and $\mathrm{P} 3 \mathrm{~b}$ components elicited by the negative distractor. Emotional distractors also elicited a posterior positivity resembling the Pd component, which might reflect the process of disengaging attention from the negative picture.

Are the same mechanisms that are at work in the $\mathrm{AB}$ also responsible for EIB? We found some striking similarities between ERP effects observed in the two paradigms. In both cases, an inability to report a target following an earlier salient input was associated with a suppression of the $\mathrm{N} 2$ and $\mathrm{P} 3 \mathrm{~b}$ components associated with the target. Our results show that emotional distractors capture attention early enough in processing to be reflected in the $\mathrm{N} 2$ component and that they disrupt attentional allocation to, and WM consolidation of, subsequently presented targets. EIB and the $\mathrm{AB}$ appear to involve the same limited-capacity mechanism that serves as a bottleneck in the processing of rapidly presented visual information. The question as to whether this overlap is complete, or whether there may yet be some unique aspect to the route through which emotional distractors disrupt target perception, awaits further investigation.

Acknowledgments This work was supported by NIH grant R03 MH091526 to S.B.M. and NSF grant BCS-1059560 to J.E.H. We wish to thank Matthew Doran, Scott McLean, and Lingling Wang for helpful discussions about this project.

\section{References}

Brainard, D. H. (1997). The Psychophysics Toolbox. Spatial Vision, 10, 433-436.

Cardinal, R. N., \& Aitken, M. R. F. (2006). ANOVA for the behavioral sciences researcher. Mahwah: Erlbaum.

Chun, M. M., \& Potter, M. C. (1995). A two-stage model for multiple target detection in rapid serial visual presentation. Journal of Experimental Psychology. Human Perception and Performance, 21(1), 109-127.

Dell'Acqua, R., Jolicoeur, P., Luria, R., \& Pluchino, P. (2009). Reevaluating encoding-capacity limitations as a cause of the attentional blink. Journal of Experimental Psychology. Human Perception and Performance, 35(2), 338-351.

Dien, J. (2010). The ERP PCA Toolkit: An open source program for advanced statistical analysis of event-related potential data. Journal of Neuroscience Methods, 187(1), 138-145.

Dux, P. E., \& Marois, R. (2009). The attentional blink: A review of data and theory. Attention, Perception, \& Psychophysics, 71(8), 16831700.

Dux, P. E., Asplund, C. L., \& Marois, R. (2009). Both exogenous and endogenous target salience manipulations support resource depletion accounts of the attentional blink: A reply to Olivers et al. Psychonomic Bulletin and Review, 16(1), 219-224.

Flaisch, T., Junghöfer, M., Bradley, M. M., Schupp, H. T., \& Lang, P. J. (2008). Rapid picture processing: Affective primes and targets. Psychophysiology, 45(1), 1-10.

Foti, D., Hajcak, G., \& Dien, J. (2009). Differentiating neural responses to emotional pictures: evidence from temporal-spatial PCA. Psychophysiology, 46(3), 521-530. 
Keil, A., Debener, S., Gratton, G., Junghöfer, M., Kappenman, E. S., Luck, S. J., ' ' Yee, C. M. (2014). Committee report: Publication guidelines and recommendations for studies using electroencephalography and magnetoencephalography. Psychophysiology, 51(1), 1-21.

Kiss, M., Grubert, A., Petersen, A., \& Eimer, M. (2012). Attentional capture by salient distractors during visual search is determined by temporal task demands. Journal of Cognitive Neuroscience, 24(3), 749-759.

Kranczioch, C., Debener, S., Maye, A., \& Engel, A. K. (2007). Temporal dynamics of access to consciousness in the attentional blink. NeuroImage, 37(3), 947-955.

Lang, P. J., Bradley, M. M., \& Cuthbert, B. N. (2001). International Affective Picture System (IAPS): Instruction manual and affective ratings (Tech. Rep. No. A-5). Gainesville: University of Florida, Center for Research in Psychophysiology.

Luck, S. J. (2011). Electrophysiological Correlates of the Focusing of Attention within Complex Visual Scenes: N2pc and Related Electrophysiological Correlates. In S. J. Luck \& E. Kappenman (Eds.), Oxford handbook of event-related potential components. New York: Oxford University Press.

Luck, S. J., \& Hillyard, S. A. (1994). Spatial filtering during visual search: Evidence from human electrophysiology. Journal of Experimental Psychology. Human Perception and Performance, 20(5), 1000-1014.

Luck, S. J., Woodman, G. F., \& Vogel, E. K. (2000). Event-related potential studies of attention. Trends in Cognitive Sciences, 4(11), 432-440.

Martens, S., \& Wyble, B. (2010). The attentional blink: Past, present, and future of a blind spot in perceptual awareness. Neuroscience \& Biobehavioral Reviews, 34(6), 947-957.

Most, S. B., Chun, M. M., Widders, D. M., \& Zald, D. H. (2005). Attentional rubbernecking: Cognitive control and personality in emotion-induced blindness. Psychonomic Bulletin and Review, 12, 654-661.

Olivers, C. N. L., van der Stigchel, S., \& Hulleman, J. (2007). Spreading the sparing: Against a limited-capacity account of the attentional blink. Psychological Research, 71, 126-139.

Pelli, D. G. (1997). The VideoToolbox software for visual psychophysics: Transforming numbers into movies. Spatial Vision, 10, 437-442.

Peyk, P., Schupp, H. T., Keil, A., Elbert, T., \& Junghöfer, M. (2009). Parallel processing of affective visual stimuli. Psychophysiology, 46(1), 200-208.

Picton, T. W., Bentin, S., Berg, P., Donchin, E., Hillyard, S. A., Johnson, R., ' ' Taylor, M. J. (2000). Guidelines for using human eventrelated potentials to study cognition: Recording standards and publication criteria. Psychophys, 37(2), 127-152.

Polich, J. (2012). Neuropsychology of P300. In S. J. Luck \& E. S. Kappenman (Eds.), Handbook of event-related potential components (pp. 159-188). New York: Oxford University Press.

Reiss, J. E., Hoffman, J. E., Heyward, F. D., Doran, M. M., \& Most, S. B. (2008). ERP evidence for temporary loss of control during the attentional blink. Naples: Poster presented at the annual meeting of the Vision Sciences Society.

Sawaki, R., \& Luck, S. J. (2010). Capture versus suppression of attention by salient singletons: electrophysiological evidence for an automatic attend-to-me signal. Attention, Perception, \& Psychophysics, 72(6), $1455-1470$.

Sawaki, R., Geng, J. J., \& Luck, S. J. (2012). A common neural mechanism for preventing and terminating the allocation of attention. The Journal of Neuroscience, 32(31), 10725-10736.

Schupp, H. T., Junghöfer, M., Weike, A. I., \& Hamm, A. O. (2003a). Attention and emotion: An ERP analysis of facilitated emotional stimulus processing. Cognitive Neuroscience and Neuropsychology, 14(8), 1107-1110.

Schupp, H. T., Junghöfer, M., Weike, A. I., \& Hamm, A. O. (2003b). Emotional facilitation of sensory processing in the visual cortex. Psychological Science, 14(1), 7-13. Elsevier.

Schupp, H.T., Flaisch, T., Stockburger, J., \& Junghöfer, M. (2006). Emotion and attention: Event-related brain potential studies. In Anders, Ende, Junghöfer, Kissler, \& Wildgruber (Eds.), Prog Brain Res, Vol. 156 pp. 31-51.

Sergent, C., Baillet, S., \& Dehaene, S. (2005). Timing of the brain events underlying access to consciousness during the attentional blink. Nature Neuroscience, 8(10), 1391-1400.

Shapiro, K., Schmitz, F., Martens, S., Hommel, B., \& Schnitzler, A. (2006). Resource sharing in the attentional blink. NeuroReport, 17(2), 163-166.

Sheppard, D. M., Duncan, J., Shapiro, K. L., \& Hillstrom, A. P. (2002). Objects and events in the attentional blink. Psychological Science, $13,410-415$.

Spencer, K. M., Dien, J., \& Donchin, E. (2001). Spatiotemporal Analysis of the Late ERP Responses to Deviant Stimuli. Psychophysiology, 38, 343-358.

Vogel, E. K., \& Luck, S. J. (2002). Delayed working memory consolidation during the attentional blink. Psychonomic Bulletin and Review, 9(4), 739-743.

Vogel, E. K., Luck, S. J., \& Shapiro, K. L. (1998). Electrophysiological evidence for postperceptual suppression during the attentional blink. Journal of Experimental Psychology. Human Perception and Performance, 24(6), 1656-1674.

Wang, L., Kennedy, B. L., \& Most, S. B. (2012). When emotion blinds: A spatiotemporal competition account of emotion-induced blindness. Frontiers Psychology: Special Topic on Emotion and Cognition, 3, 438.

Weinberg, A., \& Hajcak, G. (2011). The late positive potential predicts subsequent interference with target processing. Journal of Cognitive Neuroscience, 23, 2994-3007.

Woodman, G. F., Arita, J. T., \& Luck, S. J. (2009). A cuing study of the N2pc component: An index of attentional deployment to objects rather than spatial locations. Brain Research, 1297, 101-111. 